\section{P-292 AGING, PRODUCTIVITY AND WORK ABILITY FOR WORK IN NURSING WORKERS IN BRAZIL}

${ }^{1}$ Patrícia Baptista, Anna Telles. 'University of Sao Paulo, Brazil

\subsection{6/OEM-2021-EPI.264}

Introduction The problem of aging at work has been discussed at national and international levels, given its impact on productivity and the management of public policies on worker health.

Objective To investigate the association between chronological aging, work ability and productivity in nursing workers.

Method Analytic and cross-sectional study, with a quantitative approach, developed at a Public Hospital in the state of São Paulo, with nursing team workers aged 45 and over. Data were collected through a form containing sociodemographic and professional questions, a questionnaire on Work Ability Index and a questionnaire on work productivity.

Results 211 (79.9\%) nursing workers participated in the study. The mean age was 53 years ( $S D=4.3$ years), ranging from 45 to 71 years. The average of the Work Ability Index score was considered good, with 37.8 points, but $41.7 \%$ with a Capacity Index for inadequate work. The predominant health problems were musculoskeletal diseases. Productivity showed an average score of $24.7 \%$ for 'damage to daily activities due to health', $22.7 \%$ for 'total work injury due to health' and $21.2 \%$ for 'presentism'. There was a statistically significant association between productivity and work ability $(\mathrm{p}<0.001)$, where productivity impairment was among those with inadequate capacity (moderate and low).

Conclusion It is important to identify the profile of nursing workers in the aging phase so that the institutions could promote strategies and interventions aimed at this age group in order to prevent early retirement and promote a better longevity.

\section{P-293 A FEASIBILITY STUDY FOR DEVELOPING AN OCCUPATIONAL EXPOSURE-CONTROL INTELLIGENCE SYSTEM (OCCECIS) FOR GREAT BRITAIN}

'Ioannis Basinas, Yulia Rozanova, Andre Freitas, Damien McElvenny, Chris Keen, Yiquin Chen, Peter Baldwin, Martie van Tongeren. 'University of Manchester, United Kingdom

\subsection{6/OEM-2021-EPI.265}

Information on where occupational exposures to dangerous substances occur, how many workers are exposed, the levels of exposure, and the uptake of control measures and their effectiveness are crucial for the development and monitoring of effective interventions and exposure prevention in the workplace. The feasibility of establishing an Occupational Exposure-Control Intelligence System (OccECIS) for use in Great Britain was assessed. A scoping exercise mapped existing information of exposure tools and databases. The information sources were expanded further by input from an International Expert Advisory Committee (EAC) established to support the project in all aspects and analysed for their basic characteristics and relevance to the system. The outputs of the analysis were used together with inputs from the EAC to establish the basic conceptual framework underlying the system. This framework was converted to a set of theoretical questions relevant for the systems outputs which formed the basis for separate stakeholder and gap analyses. The outputs of these analyses were then used to develop technical solutions. The feasibility of the approach was tested on a small scale using National GB data for the construction and brick manufacturing industries. Data on exposure intensity were extracted from the National Exposure DataBase (NEDB). Prevalence of exposure was estimated by combining data from the UK census 2011, the Labour Force Survey and a previously established quantitative job exposure matrix. The results were used to illustrate examples of relevant outputs for the system. Overall, these preliminary findings suggest that it is feasible to establish such an intelligence system. This system once developed can provide leading indicators to relevant stakeholders to inform health burden and impact assessments, intervention strategies, and policy actions. A prototype of the system that will be next developed will most likely be based on the example of respirable crystalline silica.

\section{P-295 PERCEPTION OF OCCUPATIONAL EXPOSURE OF NOISE AND ITS IMPACTS ON FISH HARVESTERS' HEALTH IN NEWFOUNDLAND AND LABRADOR: A MIXED-METHOD STUDY}

'Om Prakash Yadav, Atanu Sarkar, Veeresh Gadag, Desai Shan. 'Memorial University of Newfoundland, Canada

\subsection{6/OEM-2021-EPI.266}

Introduction Noise exposure is a significant concern for fish harvesters, as it can cause serious health problems. Occupational noise exposure can result in hearing loss and non-auditory health issues such as annoyance, asthma, insomnia, cognitive disability, and diminished quality of life and wellbeing.

Objectives The aim of this study is to determine how fish harvesters in Newfoundland and Labrador perceive noise risk and to examine their experiences with noise exposure, noiserelated health problems, and barriers and challenges associated with preventing hearing loss.

Methods A mixed-methods study was conducted among NL fish harvesters. The study comprises an online questionnaire and telephone interviews. Survey tool consist of a 37-item questionnaire included noise risk perception and self-reported hearing loss questions. A semi-structured interview guide was developed to elicit information about fish harvesters' experiences with noise exposure and related health issues, as well as the obstacles and challenges associated with noise reduction and hearing loss prevention.

Results The survey results represents that an average noise risk awareness score of 2.3 to 2.9 out of 5 based on perceived benefits, barriers, and self-efficacy reflects that NL fish harvesters have a relatively positive attitude toward noise reduction and hearing loss prevention. Similarly, the noise-related perceived attitude and susceptibility score (3.9 to 4.5 ) shows that harvesters disliked the high level of noise and indicated a high risk of hearing loss. Around $62 \%$ of participants reported having hearing problems. Most participants acknowledged their workplace is noisy. There was a conflict between onboard safety and wellbeing.

Conclusions Harvesters stop wearing hearing protectors on a daily basis for safety reasons. Participants emphasized the 
importance of increasing education and awareness, training, and the use of specialized equipment in order to minimize noise exposure. Regulating onboard noise levels is necessary to avoid noise-related health problems.

\section{P-305 A MEDIA SURVEILLANCE ANALYSIS OF COVID-19 WORKPLACE OUTBREAKS IN CANADA AND THE UNITED STATES}

'Shelby Fenton, Cheryl Peters, Emma Quinn, Ela Rydz, Hugh Davies, Robert Macpherson, Chris McLeod, Mieke Koehoorn, Emily Heer. ${ }^{\top}$ Alberta Health Services, Canada

\subsection{6/OEM-2021-EPI.267}

Introduction The news media is one of the most accessible sources of information regarding COVID-19 transmission in the workplace in the absence of other occupational data. Only a few public health agencies in Canada and the United States have publically reported detailed occupation information for non-health care worker COVID-19 cases.

Objective We conducted a media surveillance analysis to identify new or emerging occupational groups at risk of exposure to the SARS-CoV-2 virus ('COVID-19 exposure').

Methods We searched the Factiva database for media articles reporting COVID-19 workplace outbreaks (February 1 December 22, 2020). Job titles were coded to the 2016 National Occupational Classification (V1.3) and industries to the 2017 North American Industry Classification System (V3.0). Occupations with COVID-19 workplace transmission identified in media articles were compared and contrasted with the same occupation in the Vancouver School of Economics (VSE) COVID Risk Tool by risk rating (seven categories between very high to very low).

Results We identified 1,111 unique COVID-19 workplace outbreaks in the media. After nurse aides, orderlies and patient service associates, industrial butchers and meat cutters, poultry preparers and related workers had the most workplace outbreaks reported in the media $(n=79)$ but were rated as medium risk occupations for COVID-19 transmission in the VSE COVID Risk Tool. Outbreaks were also reported in the media among material handlers $(n=61)$ and general farm workers $(n=28)$ but were rated medium-low risk and low risk, respectively. Outbreaks reported in the media among food and beverage services $(n=72)$ and cashiers $(n=60)$ were identified as high risk occupations in the VSE COVID Risk Tool.

Conclusion Media surveillance can identify COVID-19 workplace outbreaks and indicate transmission risk. Our results point to key determinants of health that compound the risk of COVID-19 exposure in the workplace, and highlight the importance of collecting occupation data during a pandemic.

\section{P-309 INVESTIGATING HEALTH AND OTHER CHARACTERISTICS OF MILITARY VETERANS AUTHORIZED TO RECEIVE MEDICINAL CANNABIS IN CANADA}

${ }^{1}$ Angela Czarina Mejia, Mieke Koehoorn, Hugh Davies, Amy Hall, Linda VanTil. 'University of British Columbia - Vancouver, Canada

10.1136/OEM-2021-EPI.268
Introduction Veterans Affairs Canada (VAC) has reimbursed cannabis for medical purposes (CMP) since 2008. However, to date little is known about the characteristics of Veterans authorized for CMP, and whether these differ by authorization amount.

Objectives To descriptively summarize social, health, and other characteristics of Canadian Regular Force Veterans authorized to receive CMP from Veterans Affairs Canada.

Methods A linked database of CMP authorizations was developed using VAC reimbursement files, VAC client records, and military personnel data. Analyses were limited to 13,173 Regular Force Veterans residing in Canada with an active authorization as of December 31, 2020. CMP authorization amounts (mean and categorical) were summarized by sociodemographic factors, pensionable conditions and benefits, and military service characteristics.

Results Overall, the average amount of a CMP authorization among Canadian Veterans was 3.6 grams/day. For sociodemographic characteristics, the highest average amounts were observed among Veterans who were aged 30 to 39 years $(4.2 \mathrm{~g} /$ day $)$, male $(3.7 \mathrm{~g} / \mathrm{day})$, separated/divorced/widowed $(3.8 \mathrm{~g} /$ day), and residing in the provinces of New Brunswick $(4.7 \mathrm{~g} /$ day), Newfoundland and Labrador (4.1g/day) and Manitoba (4.1g/day). For conditions documented as part of the VAC benefit process, the highest average amounts were observed among Veterans with mental health $(3.9 \mathrm{~g} /$ day) and hearing loss conditions (3.7g/day). For military service characteristics (sub-sample of 9,200 Veterans) the highest average amounts were observed among Veterans with a more recent release year, peaking in 2016 (4.2g/day); and among those who were Junior Non-Commissioned Members ( $4.0 \mathrm{~g} /$ day), had served in the army $(4.0 \mathrm{~g} / \mathrm{day})$, and released from the military involuntarily $(4.8 \mathrm{~g} /$ day $)$.

Conclusion This descriptive epidemiology provides new insights on the characteristics of a large population of veterans with medical cannabis authorizations in Canada. This will be used to inform further research on associations between CMP authorizations and wellness outcomes among military veterans.

\section{P-314 ESTABLISHING A EUROPEAN-AMERICAN POOLED COHORT OF STYRENE EXPOSED REINFORCED PLASTICS WORKERS}

${ }^{1}$ Mette Christensen, Damien McElvenny, Yvette Christopher-de Vries, loannis Basinas, Martie van Tongeren, John Cherrie, Georgia Ntani, Vanessa Cox, David Coggon, Stephen Bertke, Robert Daniels, Nicola Caranci, Stefano Mattioli, Stefania Curti, Eero Pukkala. 'Aarhus University, Denmark

\subsection{6/OEM-2021-EPI.269}

Introduction Styrene is genotoxic, an animal carcinogen, associated with lymphohematopoietic malignancies in humans, and classified as a group $2 \mathrm{~A}$ carcinogen by the IARC.

Objective To combine and harmonize existing cohorts of reinforced plastics industry workers with the aim to study exposure-response relations for styrene and subtypes of lymphohematopoietic and other malignancies as well as nonmalignant diseases.

Methods Six European cohorts included in an earlier IARC coordinated cohort and one US cohort participated. They all have been previously used in investigation of the health risks of styrene exposure. They will be updated with extended follow-up until 2019-2020 in national registries for mortality 